\title{
Research on the modelling and simulation of die casting process for A356
}

\author{
Wang Zexuan', Yang Yong ${ }^{2}$ \\ College of Mechanical Engineering, Shanghai University of Engineering Science, Shanghai, China ${ }^{1,2}$
}

\begin{abstract}
To describe the flow behaviors of semi-solid state alloy A356, two non-Newtonian constitutive equations are modeled in this paper. The CFD software PROCAST is used to simulate the process of die filling. The filling temperature is $585^{\circ} \mathrm{C}$ (solid fraction is 0.4 ). Based on the results of the simulation, it is found that the material in semi-solid state exhibits an apparently higher viscosity and thus, flows much more smoothly than the Newtonian fluid. In addition, the semi-solid metal alloy has a special die filling behavior compared with liquid filling which is of great importance in improving the quality of the final components.
\end{abstract}

Keywords: FEM simulation; ProCAST software; A356 alloy.

\section{INTRODUCTION}

In order to reduce the weight and the costs of automobiles, research and production of aluminum alloys is gaining interest rapidly in recent years. Semi-solid processing, as a near-net-shape forming technology, combines the advantages of conventional casting and forging. Aluminum alloys performed in semi-solid state exhibit a unique microstructure of globular grains suspended in the liquid metal matrix.

\section{MODELING OF THE SEMI-SOLID FLUID BEHAVIOR}

\section{A. Power Law Cut-off model}

Orgeas et al. have used the Power Law Cut-off model in the PROCAST commercial software. The model has the following form:

$$
\begin{array}{ll}
\eta=\mu_{0}\left(K \cdot \&^{n}\right. & \text { if } \& \&, \& \\
\eta=\mu_{0}(K \cdot \&)^{n} & \text { if } \& \leq \& .
\end{array}
$$

The material is assumed to be a viscous, isotropic, incompressible fluid. There is a cut-off shear-rate that is determined by the geometry of the cavity. When the shear rate exceeds the cut-off value, the material exhibits the shear thinning behavior since the de-agglomeration of the grains. However, the agglomeration of solid particles does not take place over the very short injection time. Therefore, the viscosity of the slurry will not increase after that.

The paper assumes that the viscosity is relevant to the solid fraction and shear-rate. Here we use the viscosity equation:

$$
\eta_{a}=a \exp \left(b f_{s}\right) \mathbb{R}^{\&}
$$

Where the parameters $\mathrm{a}, \mathrm{b}$ and $\mathrm{n}$ are all constant and should be fitted by the MATLAB. Thus the equation can be written as:

$$
\eta_{a}=2.720 \exp \left(6.20 f_{s}\right) \&^{0.689} .
$$

The relationship of apparent viscosity and shear-rate can be plotted and compared with the experiment results as shown in Fig. 1 (b). There is a reasonable agreement between the experiment results and the fitting results. The PLCO model can be seen as the modified version of the above equation and the parameters can be deduced then.

\section{B. Carreau-Yasuda model}

Carreau-Yasuda model is another inner viscosity model in the simulation software PROCAST. The mathematic equation is showed as follows:

$$
\eta_{a}=\eta_{\infty}+\left(\eta_{0}-\eta_{\infty}\right)\left[1+\left(\lambda \cdot \&^{a}\right]^{\frac{n-1}{a}}\right.
$$

This model assumes that the viscosity of semi-solid material can be categorized as three zones. When the shear rate states at a relatively low level, the semi-solid slurry behaves like a Newtonian fluid and the viscosity is very high, like $10^{5}-10^{7}$ Pa.s. We call this zone the first Newtonian zone and the viscosity $\eta_{0}$. To be contrary, when the shear-rate maintains in a high level, the viscosity of the semi-solid slurry is relative low and also flows like a Newtonian fluid. We call this viscosity $\eta_{\infty}$. Besides, the semi-solid slurry is a shear thinning fluid, namely, the viscosity decreases with the increasing shear-rates.

In this paper, we assume $\eta_{0}=10^{5}$ Pa.s, $a=2, n=-0.2, \eta_{\infty}$ is the Newtonian viscosity. $\lambda$ is a function of solid fraction.

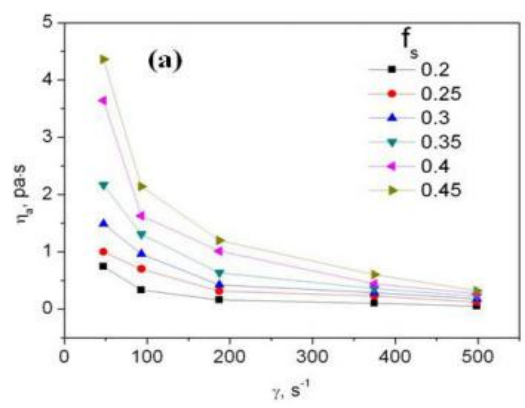




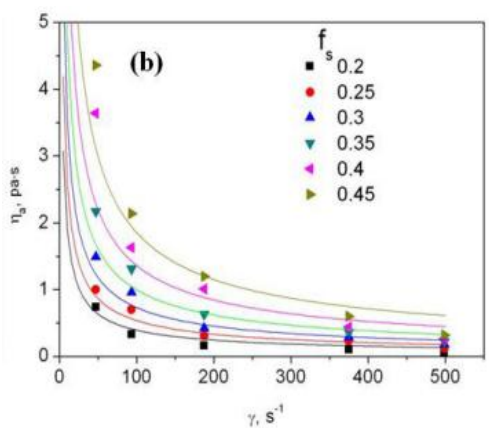

Fig.1 The relationship between apparent viscosity, shear-rate and fraction (a) Experiment data and

(b) fitting data

\section{SIMULATION OF THE SEMI-SOLID SLURRY AND DISCUSSION}

In this part, the simulation software PROCAST is used to predict the process of die filling of A356 alloy. In order to simplify the process of simulation, a general used cavity as well as its geometry size is set by Pro/E as shown in Fig.2. There exists a hole to test the flow behavior of the semi-solid. The mould is meshed then and the grid is $2 \mathrm{~mm}$. Two non-Newtonian viscosity equations are imported respectively and the boundary conditions are set. The work takes into account influence of the friction on the fluid at the surface of the mould as well as the heat transfer.
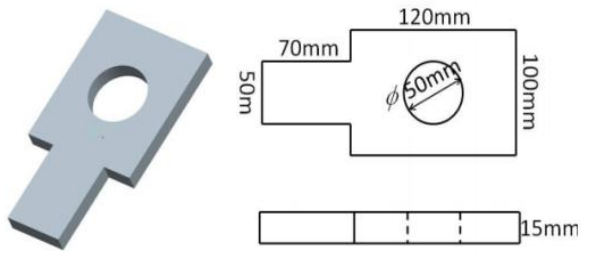

Fig.2 Three dimension mould and geometry size of mould

The simulation results are shown in Fig.3 to Fig.5. Fig.3 describes the filling process of three different viscosity models. From the Fig. 3 we can see that the Newtonian type fluid leads to the jet flow across the cavity, i.e., the material is jetting to the end of the cavity and then flowing back on itself. Defects will occur due to the splash of the material. However, the cases of Carreau-Yasuda model and Power Cut-off model are significantly different from the Newtonian fluid. The fluid flows much smoother and fills the cavity in sequence from the left to the right.

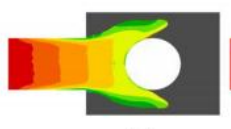

(a)

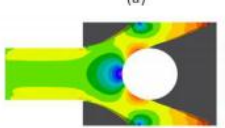

(d)

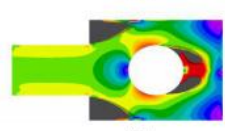

(g)

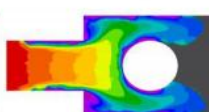

(e)

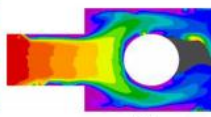

(h)
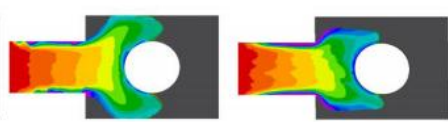

(c)

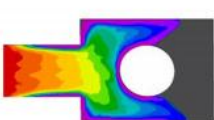

(f)

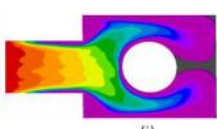

(i)
Fig.3 Comparison of filling process of the fluid of three models. (a),(d)and(g) Newtonian model,(b),(e)and(h) Carreau-Yasuda model and(c),(f) and (i) Power Law Cut-off model
This is mainly because that the viscosity of the semi-solid material is much higher than the Newtonian fluid, thus the material can fill the corner of the cavity. Furthermore, the jet flow phenomena will probably not happen at the flow front.

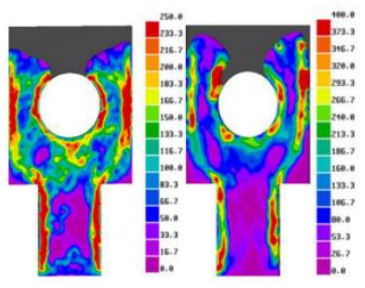

(a)

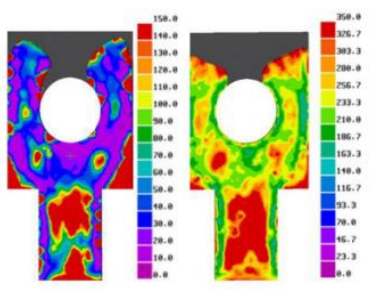

(b)
Fig.4. Comparison of shear-rate and the viscosity between the Carreau-Yasuda model and Power

Law Cut-off model. (a) shear-rate distribution and (b) viscosity distribution

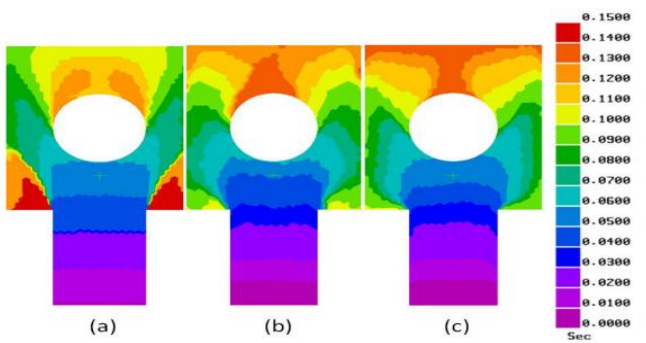

Fig.5. Comparison of filling time. (a) Newtonian model, (b )Carreau-Yasuda model and

(c) Power Law Cut-off model.

Fig.4. shows the distribution of the shear-rate and viscosity of semi-solid slurry. From the figure, we can see the fluid in the boundary sections of the cavity is more viscous which is mainly because that the frictions with the die surface increases the shear-rate among the fluid. The viscosity of the semi-solid slurry decreases due to the shear thinning behavior. However, in inner sections the influence of the shear is little so the viscosity is higher.

Fig.5. represents the filling time of the Newtonian fluid and semi-solid slurry. No apparent differences can be found comparing (a), (b) with (c). Therefore, though the flow of the semi-solid slurry is much steady and smoother, the efficiency of the production of semi-solid slurry may not drop much. From the Figure, it can be identified again that the filling time lasts only fractions of one second, so the accurate predicting of the filling process is of great importance.

\section{CONCLUSION}

Carreau-Yasuda model and Power Law Cut-off model are investigated and the parameters in the above equations have been predicted. In order to understand the flow behaviors, two constitutive equations with a Newtonian fluid are simulated by the numerical simulation software PROCAST. From the simulation results, it can be concluded as follows: (1)The behavior of semi-solid fluid is typical non-Newtonian fluid. Semi-solid slurry presents the shear-thinning behavior at the isothermal conditions. The viscosity decreases apparently when the shear-rate increases. 
(2)There exists a significant difference between conventional liquid processing and semi-solid processing over the filling pattern. Due to the higher viscosity of semi-solid slurry, the flow is smoother than the Newtonian fluid. This helps reducing the possibility of gas inclusion and overlap on the final components and thus, improving the quality of the final components.

\section{REFERENCES}

[1] Orgeas, I., Gabathuler, J.P., Imwinkelried, T.H., 2003. Modelling of Semi-solid Processing Using a Modified Temperature-dependent Powerlaw model. Modelling and Simulation in Materials Science and Engineering, 2003(11),553-574.

[2] Zhang, Y.J., Mao, W.M., Zhao, Z.D., Liu, Z., 2006. Rheological Behavior of Semi-solid A356 Aluminum Alloy at Steady State. ACTA Metallurgica Sinica, 42(2), 163-166.

[3] Lin, W.J., Zhao, S.D., Tao, W.L., Zhao, C.W, Wang, J.F., 2012. Reasonable Processing Parameters of Squeeze Casting Semi-solid A356Aluminum Alloy Connecting Rod. Special Casting and Nonferrous Alloys, 32(5),438-442. 